received treatment with cytosine - arabinoside (ara-C) based consolidation and 26 patients received treatment with anthracycline based therapy (MACE/MIDAC). 9 patients (41\%) in the araC group relapsed and 3 of these patients subsequently died. In only 1 patient this was secondary to resistant disease. Of the 9 patients who relapsed 6 had adverse cytogenetics. Within the group of patients treated with MACE/MIDAC consolidation, 14 relapsed (54\%) and of these, 10 patients subsequently died. 6 of the 14 relapsed patients had adverse cytogenetic profiles. No patient within the MACE/ MIDAC group had treatment changed due to cardiotoxicity. Chisquared analysis of death rates identified a $\mathrm{p}$ value $<0.1$, but more than 0.05 .

Conclusions The outcome analysis indicates that there is no significant difference between rates of relapse or death in the 2 groups of patients.

\section{TEN-YEAR FOLLOW-UP OF INFANTS WITH SYMPTOMATIC AND ASYMPTOMATIC CONGENITAL CYTOMEGALOVIRUS INFECTION}

doi:10.1136/archdischild-2012-302724.0261

${ }^{1} M G$ Capretti, 'C Marsico, 'M Spinelli, 'M De Angelis, ${ }^{2} \mathrm{~T}$ Lazzarotto, ${ }^{2} \mathrm{~L}$ Gabrielli, ${ }^{2} \mathrm{~A}$ Chiereghin, ${ }^{2} \mathrm{G}$ Piccirilli, ${ }^{2} \mathrm{E}$ Petrisli, 'L Corvaglia, ${ }^{3} \mathrm{M}$ Lanari, ${ }^{1} \mathrm{G}$ Faldella. ${ }^{1}$ Department of Obstetrical, Gynaecological and Paediatric Sciences, Operative Unit of Neonatology; ${ }^{2}$ Department of Haematology, Oncology and Laboratory Medicine, Operative Unit of Microbiology and Virology, St. Orsola-Malpighi University Hospital, University of Bologna, Italy, Bologna; ${ }^{3}$ Department Paediatrics and Neonatology Unit, S. Maria della Scaletta Hospital, Imola, Italy

Background and Aims Congenital Cytomegalovirus (CMV) infection can lead to neurological sequelae and sensorineural hearing loss (SNHL). To correlate clinical, auditory and neuroimaging findings in the neonatal period to long-term outcome in congenitally CMV-infected infants.

Methods Congenitally CMV-infected infants born between 2001 and 2011 were clinically evaluated and underwent cranial Ultrasound (cUS), cerebral Magnetic Resonance Imaging (cMRI), fundoscopy examination and auditory brainstem response (BAER) in the neonatal period. Both symptomatic and asymptomatic infants were followed prospectively to assess physical growth, neurological, visual and audiological outcome.

Results Forty-two infants were evaluated. Six of 42(14.2\%) infants had symptoms/signs at birth: microcephaly (3), petechiae (4), thrombocytopenia (3), hepatosplenomegaly (3), jaundice (1), elevated serum transaminases (1). Two cases of chorioretinitis and 4 cases of abnormal BAER were found in the neonatal period. cUS demonstrated pathological findings in $6 / 42(14.2 \%)$ infants: ventriculomegaly (4), pseudocysts (3), calcifications (3), cerebellar hypoplasia (1). cMRI showed abnormalities in 10/42(23.8\%) infants: pseudocysts (3), white-matter lesions (7), lissencephaly (1), ventriculomegaly (4), calcifications (2), cerebellar hypoplasia (2). At follow-up (mean duration $43 \pm 18$ months) 8/42(19\%) infants showed SNHL and 8/42(19\%) showed impaired psychomotor development. The composite outcome (SNHL and/or neurodevelopmental sequelae) was poor in 9/42(21.4\%) infants. Neonatal findings in infants with an adverse outcome were: clinical signs (5/9), abnormal BAER (2/9), abnormal cUS (5/9), abnormal cMRI (9/9). Symptomatic infants received antiviral treatment.

Conclusions In our series $21.4 \%$ of congenital CMV infected infants had one or more sequelae at follow-up evaluations. A pathological neuroimaging at birth was the most sensitive predictor of long-term sequelae.

\section{WHETHER MULTI-RESISTANT STRAINS OF NON-TYPHOID SALMONELLOSIS AFFECT CLINICAL MANIFESTATIONS AND OUTCOMES IN CHILDREN?}

doi:10.1136/archdischild-2012-302724.0262
1.2IF Huang, 'HH Hu, 1,2MF Chang, 2,3YS Chen, 'SK Sheu, 'KS Hsieh, 1,2CC Chiou. ${ }^{1}$ Pediatrics, Kaohsiung Veterans General Hospital, Kaohsiung; ${ }^{2}$ National Yang-Ming University, Taipei; ${ }^{3}$ Medicine, Kaohsiung Veterans General Hospital, Kaohsiung, Taiwan R.O.C.

Objective The aim of the study was to investigate whether multiresistant strains of non-typhoid salmonellosis affect clinical manifestations and outcomes in children.

Methods Between 1996 and 2009, children with nontyphoid salmonellosis admitted to Kaohsiung Veterans General Hospital, Taiwan were enrolled. An organism was considered multi-resistant if resistant to $\geq 2$ agents. Fecal excretion time was defined as the timeframe of the first positive stool culture and the first of two consecutive negative results. The demographic, laboratory data and clinical outcomes were compared between the patients infected with nontyphoid Salmonella susceptible to all antibiotics (susceptible group) and those infected with multi-resistant strains (resistant group).

Results 764 patients were enrolled; 318 were characterized as the susceptible group; 329 were the resistant group. Compared with susceptible group, the patients of resistant group were younger (32.36 vs 23.65 months of age); demonstrated significantly more bloody stool; higher white blood cells; longer total fever days and hospital stay (38.4\% vs $49.2 \%$; 9,538 vs 10,205/Cumm; 5.75 vs 6.66 days; 8.38 vs 9.49 days, respectively). However, there were no statistically significant differences in C-reactive protein (7.74 vs $7.82 \mathrm{mg} /$ $\mathrm{dl}$ ), occurrence of bacteremia (15.04\% vs $14.57 \%$ ), rates of antibiotics used $(67.30 \%$ vs $70.21 \%)$, complications (3.77\% vs $2.43 \%$ ) and fecal excretion time [19.25 ( $=20)$ vs $11.69(\mathrm{~N}=16)$ days].

Conclusion The children with multi-resistant non-typhoid salmonellosis had more severe clinical manifestations and worse outcomes in terms of bloody stool, leukocytosis, fever days and hospital stay. Antibiotics use in humans and animals should be weighed against the development of resistance.

\section{RSV HOSPITALIZATION IN CYSTIC FIBROSIS IN THE CANADIAN REGISTRY OF SYNAGIS ${ }^{\circledR}$ (CARESS) FOLLOWING PROPHYLAXIS (2005-2011)}

doi:10.1136/archdischild-2012-302724.0263

'B Paes, ${ }^{2} \mathrm{~A} \mathrm{Li},{ }^{3} \mid \mathrm{M}$ Mitchell, ${ }^{2} \mathrm{KL}$ Lanctot. 'Paediatrics, McMaster University, Hamilton ${ }^{2}$ Medical Outcomes and Research in Economics (MORE), Sunnybrook Health Sciences Centre, Toronto, ON; ${ }^{3}$ Paediatrics (Respirology Division), University of Calgary, Calgary, $A B$, Canada

Background and Aims The Canadian Registry of Synagis ${ }^{\circledR}$ (CARESS) tracks palivizumab utilization and respiratory syncytial virus (RSV) hospitalizations in high-risk infants including 186 with cystic fibrosis (CF). This study compares respiratory illness (RI) and RSV positive hospitalization (RSVH) rates in CF infants versus:

1. those with other underlying medical disorders (MD) and

2. those who meet standard indications for RSV prophylaxis (SD).

Methods A prospective, observational registry of infants from 30 sites who received $\geq 1$ dose of palivizumab during the 2005-2011 RSV seasons. Palivizumab utilization and RI outcomes were collected monthly over each individual season.

Results 10,452 infants were enrolled (CF: 186, 1.8\%; MD: 1378, $13.2 \%$; SD: $8888,85.0 \%)$. There were significant differences $(p<0.05)$ between the three groups in gestational and enrolment ages, weight at birth and at enrolment and proportions of: Caucasians, siblings, multiple births, daycare attendance, smokers in the household, $>5$ people in the household, immediate family history of atopy, and complexity of neonatal course. Infants with CF had a lower RI hospitalization rate than the $\mathrm{MD}(4.3 \%$ versus $11.0 \%, \mathrm{p}=0.003)$ but not the SD group $(5.8 \%, p=0.522)$. For RSVH rate, CF infants were not significantly different from the $\mathrm{MD}(0.72 \%$ versus $2.46 \%, \mathrm{p}=0.227)$ 